\title{
A semi-analytic power balance model for low (L) to high (H) mode transition power threshold
}

\author{
R. Singh, ${ }^{1,2, a)}$ Hogun Jhang, ${ }^{1}$ P. K. Kaw,${ }^{2}$ P. H. Diamond, ${ }^{1,3,4}$ H. Nordman, ${ }^{5}$ C. Bourdelle, ${ }^{6}$ \\ and A. Loarte ${ }^{7}$ \\ ${ }^{1}$ WCI Center for Fusion Theory, National Fusion Research Institute, Daejeon 305-333, South Korea \\ ${ }^{2}$ Institute for Plasma Research, Bhat Gandhinagar 2382 428, India \\ ${ }^{3}$ Center for Momentum Transport and Flow Organization, University of California, San Diego, \\ California 92093, USA \\ ${ }^{4}$ Center for Astrophysics and Space Sciences, University of California, San Diego, \\ 9500 Gilman Dr., La Jolla, California 92093-0424, USA \\ ${ }^{5}$ Department of Earth and Space Sciences, Chalmers University of Technology, SE-412 96 Göteborg, Sweden \\ ${ }^{6}$ Euratom-CEA Association, CEA/DSM/DRFC, CEA Cadarache F-13108 Saint-Paul-Lez-Durance, France \\ ${ }^{7}$ ITER Organization, Route de Vinon Sur Verdon, A. 13115 Saint Paul Lez Durance, France
}

(Received 14 April 2014; accepted 23 May 2014; published online 9 June 2014)

We present a semi-analytic model for low $(\mathrm{L})$ to high $(\mathrm{H})$ mode transition power threshold $\left(P_{t h}\right)$. Two main assumptions are made in our study. First, high poloidal mode number drift resistive ballooning modes (high- $m$ DRBM) are assumed to be the dominant turbulence driver in a narrow edge region near to last closed flux surface. Second, the pre-transition edge profile and turbulent diffusivity at the narrow edge region pertain to turbulent equipartition. An edge power balance relation is derived by calculating the dissipated power flux through both turbulent conduction and convection, and radiation in the edge region. $P_{t h}$ is obtained by imposing the turbulence quench rule due to sheared $E \times B$ rotation. Evaluation of $P_{t h}$ shows a good agreement with experimental results in existing machines. Increase of $P_{t h}$ at low density (i.e., the existence of roll-over density in $P_{t h}$ vs. density) is shown to originate from the longer scale length of the density profile than that of the temperature profile. C 2014 AIP Publishing LLC. [http://dx.doi.org/10.1063/1.4882256]

\section{INTRODUCTION}

Elucidating the physics of the low (L)- to high $(\mathrm{H})$ mode transition is a long standing problem since its first discovery at ASDEX. ${ }^{1}$ The H-mode is characterized by the presence of an edge pressure pedestal (i.e., edge transport barriers) and yields high fusion performance. This is of particular importance to ITER which aims at realization of high fusion gain deuterium-tritium (DT) plasmas with $H_{98} \geq 1$. Here, $H_{98}$ is defined as the ratio of energy confinement time $\left(\tau_{E}\right)$ to the energy confinement time scaling $\left(\tau_{98}\right)$ whose expression is given $b^{2}$

$$
\begin{aligned}
\tau_{98}(\text { sec })= & 0.0562\left[I_{p}(M A)\right]^{0.93}\left[B_{T}(T)\right]^{0.15}[P(M W)]^{-0.69} \\
& \times\left[n\left(10^{19} m^{-3}\right)\right]^{0.41}[M(A M U)]^{0.19} \\
& \times[R(m)]^{1.97} \epsilon^{0.58} \kappa^{0.78}
\end{aligned}
$$

where $I_{p}$ is the plasma current, $B_{T}$ the toroidal field at major radius $R, P$ the power, $n$ the plasma density, $M$ the average ion mass, $\epsilon$ the inverse aspect ratio, and $\kappa$ the elongation. Units for physical quantities are indicated in parentheses.

$\mathrm{H}$-mode operation in ITER is also planned in the nonactive (hydrogen $(\mathrm{H})$ and helium $(\mathrm{He})$ ) and the DD phases to characterize $\mathrm{H}$-mode plasmas in ITER-scale experiments and to develop reliable edge localized modes (ELMs) control schemes well before the D-T operation phase. Key dynamical processes involved in the $\mathrm{L}-\mathrm{H}$ transition physics have

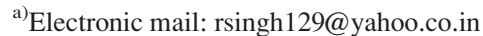

been developed for the past two decades. ${ }^{3}$ The most important concept for L-H transition physics may be the suppression/reduction of turbulence by $E \times B$ flow $^{4,5}$ and subsequent formation of a transport barrier due to the positive feedback between mean $E \times B$ shear and turbulence suppression. Recent theories and experiments highlight the role of zonal flow $^{6,7}$ as a trigger of the L-H transition. ${ }^{8-10}$

At present, however, there is still a gap between this success of microscopic understanding of $\mathrm{L}-\mathrm{H}$ transition physics and its connection to macroscopic predictive capability. A notable example of this gap is the lack of theorybased models giving a reliable, quantitative prediction of the L-H transition power threshold $\left(P_{t h}\right)$. Given the absence of such a model, experimentalists have developed empirical scaling relations which were derived based on a statistical analysis of an experimental database in present tokamak experiments. A recent study in this line shows that $P_{t h}$ depends primarily on average plasma density $(\bar{n})$, toroidal magnetic field $\left(B_{T}\right)$, and plasma surface area $(S)^{11}$

$$
P_{t h}=0.0488\left[\bar{n}\left(10^{20} m^{-3}\right)\right]^{0.717}\left[B_{T}(T)\right]^{0.803}\left[S\left(m^{2}\right)\right]^{0.941} .
$$

Equation (1) successfully reproduces experimental results of $P_{t h}$ for high density regime. However, deviations of $P_{t h}$ from the prediction of this empirical law have been observed in experiments, most notably, the increase of $P_{t h}$ below a "roll-over" density $\left(n_{\text {min }}\right)^{12-15}$ (i.e., $P_{\text {th }} \simeq \bar{n}^{-\alpha}$ with $\alpha>0$, when $\bar{n} \leq n_{\text {min }}$ ). In most of the machines, the roll-over density is observed to be in the range $0.1-0.3 \times 10^{20} \mathrm{~m}^{-3}$. One 
exception for this trend is the Alcator C-Mod tokamak for which $n_{\text {min }}$ is much higher with $n_{\text {min }}=0.8-1.2 \times 10^{20} \mathrm{~m}^{-3}$. Another omission in Eq. (1) is the isotope scaling, which shows $P_{t h} \sim A_{i}^{-\beta}$, with $\beta \approx 1.0$, where $A_{i}$ is the effective atomic mass, deduced from the comparison of $P_{t h}$ for hydrogen, DD and DT discharges. ${ }^{16}$ In the context of ITER operation which plans to obtain $\mathrm{H}$-mode in non-active phase (i.e., using $\mathrm{H}$ or He discharges), this is also an issue due to the possible limitation of available power. A recent paper summarizes experimental results of multi-machine assessment on $P_{t h}$ for $\mathrm{H}, \mathrm{He}$, and D plasmas. ${ }^{17}$ The power threshold for $\mathrm{He}, P_{t h}^{\mathrm{He}}$, shows a rather large variation ranging $1.0 \leq P_{t h}^{H e} / P_{t h}^{D} \leq 1.8,{ }^{17}$ implying the ambiguity of the isotope scaling for $\mathrm{He}$ discharges. Here, $P_{t h}^{D}$ is $P_{t h}$ for deuterium discharges.

In these regards, it is necessary to have a physics-based model that can predict $P_{t h}$ in terms of tokamak discharge parameters. Such a model necessarily should reproduce experimental features mentioned above, as well as the empirical scaling law in a statistically reliable region. The primary purpose of this work is to develop a theory-based semi-analytic expression of $P_{t h}$ which give a quantitative prediction of $P_{t h}$. To do so, we develop a model based on an edge power balance relation. One may regard our work as an "intermediate step" bridging the gap between the empirical scaling law and a complete theoretical model taking into account the full microphysical dynamics of turbulence.

The basic idea of our power balance model came from the observation of how a star maintain its equilibrium temperature profile given the energy dissipation at a narrow, outermost boundary region. Core temperatures of the Sun and other stars adjust themselves such that the energy generation in the core balances the radiative capacity of the outermost radiative zone. ${ }^{18}$ Then, the intermediate convective zone adjusts itself so as to transport all this energy flux. In a tokamak, the energy shedding capacity of the outermost edge region will be determined by radiation loss and turbulent transport (both convection and conduction) in that region. Then, based on the analogy to the Sun, we may expect that the core temperature of a tokamak plasma will increase rapidly (i.e., L-H transition occurs) when energy shedding capacity at the edge region diminishes by some reasons, such as due to either the suppression of edge turbulence or the reduction of radiative loss, depending on the relative strength of the two processes (typically turbulent transport will be much higher in normal tokamak operation). This may result in the formation of a sharp edge temperature gradient, which is accelerated by the positive feedback between the mean $E \times B$ flow and turbulent suppression.

This paper is organized as follows: In Sec. II, we derive $P_{\text {th }}$ from an edge power balance relation. We assume that power flux coming out of the core is transported by turbulent conduction and convection or dissipated by impurity radiation in a fixed narrow edge layer. To analytically evaluate the amount of turbulent heat transport in a narrow edge layer, we make two assumptions. First, we assume that the high poloidal mode number drift resistive (high- $m$ DRBM) ${ }^{19-22}$ is the dominant turbulence driver leading to the turbulent heat transport prior to L-H transition. High- $m$ DRBM driven turbulence in edge and scrape-off layer (SOL) regions has been shown in numerical simulations $s^{21,23,24}$ and some experiments. $^{25,26}$ Second, the turbulent equipartition (TEP) ${ }^{35-37}$ process is assumed to prevail in pre-transition L-mode states. Then, we apply the conventional turbulence quench rule, $\omega_{E \times B} \geq \gamma_{\text {lin }}$, where $\omega_{E \times B}=\partial v_{E \times B} / \partial r$ and $\gamma_{\text {lin }}$ is the linear growth rate of the high-m DRBM, to obtain transition condition, hence $P_{t h}$. The assumption of the prevalence of TEP prior to L-H transition leaves one numerical factor to be determined empirically from experimental data. Section III is devoted to the application of the semi-analytic formula developed in Sec. II to existing devices and ITER. The power threshold formula is found to reproduce the characteristic features observed in various devices and shows a reasonable agreement in terms of the values of minimum $P_{t h}$ and the roll-over density. Prediction of $P_{t h}$ for ITER shows that reduction of edge density is a key to ensure H-mode access in high density regime. We conclude our paper in Sec. IV with a brief summary of main results and some discussion.

\section{A POWER BALANCE MODEL FOR L-H TRANSITION}

We begin our analysis from a postulate that a narrow region in the vicinity of the last closed flux surface, which is named as the edge dissipation layer (EDL), is responsible for dissipation of energy coming from the core through turbulent conduction and convection, and radiative processes. The width of EDL is the order of electron temperature scale length, which is typically $2 \%-5 \%$ of the minor radius $(a)$, i.e., $\Delta_{R} / L_{T e} \sim 1$, where $\Delta_{R}$ is the EDL width and $L_{T_{e}}$ $=-\nabla T_{e} / T_{e}$ is the electron temperature scale length.

In typical gas-puffed L-mode discharges, neutral particles play an important role in determining edge density profiles. For instance, the strong correlation of neutral penetration depth with the shape of edge density profile has been reported previously. ${ }^{27,28}$ The density profile often shows a "pedestal-like" structure even before L-H transition. ${ }^{28}$ The density scale length $\left(L_{n}=-n / \nabla n\right)$ is then typically smaller than $L_{T_{e}}$. In this case, the atomic processes such as ionization and charge exchange will prevail and determine density profile in EDL. ${ }^{29,30}$ One can then evaluate $L_{n}$ in EDL from an one-dimensional model of neutrals and plasma particles under the assumption of edge recycling factor of unity, resulting in $^{30}$

$L_{n} \simeq 1 / n \sigma_{*}, \quad \sigma_{*}=\sqrt{m_{i}\left(\kappa_{i}+\kappa_{c x}\right) \kappa_{i} / T_{e}} \approx 3.6 \times 10^{-19} \mathrm{~m}^{2}$.

Here, $\kappa_{i}$ and $\kappa_{c x}=\langle\sigma \nu\rangle_{c x}$ are the rate coefficients for ionization and charge exchange processes, respectively. We use SI units throughout the paper, except for the electron temperature and power for which keV and MW are used, respectively, unless otherwise specified.

The radiative power inside EDL, $P_{\text {rad }}$, can be written as

$$
P_{\text {rad }}=n_{I} n R_{T} V
$$

where $R_{T}$ is the volumetric radiation, ${ }^{31} V=S \Delta_{R}$ the volume of EDL with $S=4 \pi^{2} R a \sqrt{\left(1+\kappa^{2}\right) / 2}$ the surface area, $R(a)$ 
and $\kappa$ the major (minor) radius and elongation, respectively, and $n_{I}=\sum_{z} n_{z}$ with $n_{z}$ denoting the density of impurity ions in the ionization state $z$ at a given temperature. In fact, $n_{I}$ and $R_{T}$ are complicated functions of electron temperature ${ }^{31}$ whose values should be provided from experimental measurements. In this study, however, we treat $R_{T}$ and $n_{I} \approx f_{Z} n$ (where $f_{Z}$ is the impurity fraction) as constants in the sense that an average over a narrow edge region would yield fairly fixed values for them (for given plasma facing materials and wall conditioning method) regardless of plasma discharge parameters. Normalizing $R_{T}, n$, and $f_{Z}$ by $10^{-39} \mathrm{MW} \cdot \mathrm{m}^{3}$, $10^{20} \mathrm{~m}^{-3}$, and $10^{-2}$, respectively, one can express $P_{\text {rad }}$ in MW unit

$$
\begin{aligned}
P_{\text {rad }}(\mathrm{MW})= & 0.1 \times f_{Z}\left(10^{-2}\right)\left[n\left(10^{20} \mathrm{~m}^{-3}\right)\right]^{2} \\
& \times R_{T}\left(10^{-39} \mathrm{MW} \cdot \mathrm{m}^{3}\right) S \Delta_{R},
\end{aligned}
$$

where normalized units are indicated in corresponding physical quantities.

To make any further analytical progress, it is necessary to postulate on the turbulent process occurring in EDL. Until now, there is no generally accepted theory in tokamak edge turbulence. In this study, we hypothesize that high poloidal mode number drift resistive ballooning mode (high-m DRBM $)^{19-22}$ is a dominant turbulent mode, which is responsible for the turbulent power loss in EDL prior to $\mathrm{L}-\mathrm{H}$ transition (i.e., when edge temperature is relatively low). This is one of main assumptions in this work, the justification of which should be made based on the comparison of our final results and experimental data. Recent gyrokinetic simulations using the GENE code indeed find that resistive ballooning modes are unstable around the pedestal formation region prior to L-H transition. ${ }^{24}$

High-m DRBMs have similar growth rates to ideal ballooning modes even when $\beta$ is less than the critical $\beta$, $\beta_{c} \approx \beta\left(1+\tau_{i} / Z\right) q^{2} R / L_{n}$. Here, $\beta=2 \mu_{0} P / B^{2}$, with $P$ the total pressure, $q=r B_{T} / R B_{p}$, with $B_{T}$ and $B_{p}$ are toroidal and poloidal magnetic fields, respectively, $\tau_{i}=T_{i} / T_{e}$, and $Z$ is the ionic charge. The linear growth rate $\left(\gamma_{\text {lin }}\right)$ of the high-m DRBM is given by ${ }^{21,22}$

$$
\gamma_{\text {lin }}=\sqrt{\frac{2 c_{s H}^{2}\left(1+\tau_{i} / Z\right)}{R L_{n} A_{i}}}
$$

where $c_{s H}=\sqrt{T_{e} / m_{H}}=3.09 \times 10^{5} T_{e}^{1 / 2}(\mathrm{~m} / \mathrm{s})$ is the thermal speed of hydrogen ions and $A_{i}$ is the atomic mass.

The radial correlation length of high-m DRBM is determined by balancing the compression of the polarization current and the parallel electron current. Specifically, the relation

$$
k_{\perp}^{2} \rho_{s}^{2} \gamma_{l i n} \approx \chi_{e} k_{\|}^{2}
$$

yields the approximate radial correlation length $\left(L_{0}\right)$ of high-m DRBM ${ }^{21}$

$$
L_{0} \approx\left[\frac{q^{2}}{\hat{s}^{2}} \frac{\nu_{e} \rho_{s H} R}{2 \Omega_{e}}\right]^{1 / 2}\left[\frac{2 R\left(1+\tau_{i} / Z\right) A_{i}}{L_{n}}\right]^{1 / 4}
$$

In Eqs. (6) and (7), $k_{\perp}$ and $k_{\|}$represent wavenumber of high-m DRBM in perpendicular and parallel direction, respectively, $\nu_{e}$ denotes the electron-ion collision frequency for singly charged ions, the numerical value of which is given by $\nu_{e}^{-1}=\tau_{e}=6.4 \times 10^{14} T_{e}(\mathrm{keV})^{3 / 2} / n\left(\mathrm{~m}^{-3}\right)$, with $n$ the edge plasma density, $\rho_{s H}=c_{s H} / \Omega_{i}, \Omega_{e}$ is the electron gyro-frequency and $\hat{s}=d \ln q(r) / d \ln r$.

In the quasilinear theory of DRBM-driven turbulent transport, particle and thermal diffusivities are approximately equal. One can then use the mixing length argument to evaluate them to obtain

$$
\begin{aligned}
D_{\text {turb }} & \approx \chi_{e}=\gamma_{l i n} L_{0}^{2} \approx\left(1+\tau_{i} / Z\right)(q / \hat{s})^{2}\left(R / L_{n}\right) \nu_{e} \rho_{e}^{2} \\
& =\left(1+\tau_{i} / Z\right)(q / \hat{s})^{2}\left(R / L_{n}\right) \frac{1.79 \times 10^{-23}}{n T_{e}^{1 / 2}}\left(\frac{n}{B_{T}}\right)^{2} .
\end{aligned}
$$

Here, $\rho_{e}=\sqrt{m_{e} T_{e}} / e B_{T}=1.07 \times 10^{-4} T_{e}^{1 / 2} / B_{T}(m)$ is the electron gyroradius and we use $\ln \Lambda=17$ and $\mathrm{keV}$ units for $T_{e}$. We remark that Eq. (8) does not necessarily imply the same profiles for density and temperature because the edge density profile is determined by the combination of turbulent diffusion, particle pinch, and neutral penetration depth.

At this point, it is appropriate to introduce fundamental assumptions in our study. We assume that the pre-transition L-mode edge profile and turbulent diffusivity within EDL are pertinent to turbulent equipartition (TEP) ${ }^{35-37}$ with high$m$ DRBM as the dominant turbulence driver. The basic idea of the TEP theory is that turbulent mixing leads to the homogenization of magnetically weighted, locally conserved quantities. The result of this homogenization is $n / B_{T} \sim$ const on a surface. ${ }^{36}$ Naulin et al. shows that the relation $n / B_{T} \sim$ const is well established from self-consistent, flux-driven TEP simulations with electrostatic pressure gradient driven interchange turbulence in slab geometry. ${ }^{36}$ Note that the characteristics of high- $m$ DRBM and interchange turbulence are almost identical. Under this assumption, we may treat $\left(n / B_{T}\right)^{2}$ term in Eq. (8) as a constant, denoted by $C_{T E P}$, representing the TEP effect.

Under these assumptions, the turbulent power through EDL becomes

$$
\begin{aligned}
P_{\text {turb }}= & \left(T \Gamma_{n}+n \Gamma_{e}\right) S \\
\approx & 1.79 \times 10^{-23} C_{T E P}\left(1+\tau_{i} / Z\right)(q / \hat{s})^{2} T_{e}^{1 / 2}\left(n \sigma_{*}\right)^{2} \\
& \times\left[1+\frac{1}{n \sigma_{*} L_{T}}\right] R S,
\end{aligned}
$$

which can be re-written in terms of MW units

$$
\begin{aligned}
P_{\text {turb }}(\mathrm{MW})= & 0.286 \times 10^{-4} C_{T E P}\left(1+\tau_{i} / Z\right) T_{e}^{1 / 2}(\mathrm{keV}) \\
& \times n^{2} \sigma_{*}^{2}\left(1+\frac{1}{n \sigma_{*} L_{T}}\right) R S
\end{aligned}
$$

where $\sigma_{*}$ is defined in Eq. (2). To obtain Eq. (9), we use the Fick's law for the particle and thermal flux, $\Gamma_{n}=-D \partial n / \partial r$ and $\Gamma_{e}=-\chi_{e} \partial T_{e} / \partial r$, respectively, and assume that $q / \hat{s}$ is constant and order of unity, $q / \hat{s} \simeq 1$. This is a reasonable approximation in many tokamak discharges with $q_{95} \geq 2.5$ and divertor configuration. 
To evaluate the threshold edge electron temperature beyond which L-H transition occurs, we employ the conventional turbulence suppression criterion. ${ }^{4}$ Thus, L-H transition occurs when the $E \times B$ shearing rate exceeds the inverse of turbulence auto-correlation time $\left(\tau_{c}\right)$, i.e., $\omega_{E \times B}=\partial\left\langle V_{E \times B}\right\rangle$ $/ \partial r>\tau_{c}^{-1}$, where $\tau_{c}^{-1} \approx \gamma$ in the quasi-linear limit. One can obtain $\omega_{E \times B}$ from a radial force balance relation.

There are three main contributors to $\omega_{E \times B}$ involved in L-H transition: (1) the ion diamagnetic component coming from the ion pressure gradient $\left(\nabla P_{i}\right),(2)$ the poloidal zonal flow from poloidal Reynolds stress, and (3) the component driven by an ion parallel flow gradient $\left(\nabla V_{\|}\right)$. In nominal $\mathrm{L}-\mathrm{H}$ transition involving the formation of the type-I ELMy $\mathrm{H}$-mode, it has been known that components (1) and (2) are important with $\nabla P_{i}$ being the dominant, while (3) plays an important role in core transport barrier formation. ${ }^{9,32,33}$ Poloidal zonal flows play a crucial role in the early phase of L-H transition by suppressing background turbulence, hence triggering the $\mathrm{L}-\mathrm{H}$ transition. The actual $\mathrm{L}-\mathrm{H}$ transition, however, occurs when the $\nabla P_{i}$ component becomes dominant and provides the positive feedback for further steepening of the pressure gradient, as predicted by the two predator-one prey model ${ }^{34}$ and recently confirmed in DIII-D experiments. ${ }^{9}$ In this regard, we can neglect $\nabla V_{\|}$and zonal flow contributions to $\omega_{E \times B}$ during the positive feedback period of L-H transition.

The $\nabla P_{i}$ component can be further divided into density $(\nabla n)$ and temperature gradient $\left(\nabla T_{i}\right)$ driven components. Normally, L-H transition involves the development of a strong density gradient, as manifested by a sudden drop in $\mathrm{H}_{\alpha} / \mathrm{D}_{\alpha}$ signal and the increase of the edge plasma density. Thus, the pressure gradient is mostly dominated by the density gradient component during the $\mathrm{L}-\mathrm{H}$ transition.

Based on above discussions, one can safely assume that $\omega_{E \times B}$ comes mostly from the density gradient

$$
\omega_{E \times B} \approx \frac{T_{i}\left(\nabla_{r} n\right)^{2}}{e Z B_{T} n^{2}}=\frac{\tau_{i} T_{e}}{e Z B_{T} L_{n}^{2}}>\sqrt{\frac{2 c_{s H}^{2}\left(1+\tau_{i} / Z\right)}{R L_{n} A_{i}}},
$$

during L-H transition. Then, substitution of $L_{n} \approx 1 / n \sigma_{*}$ to Eq. (10) results in an expression for the threshold electron temperature

$$
\begin{aligned}
& {\left[T_{e}(\mathrm{keV})\right]^{1 / 2} \geq 3.09 \times 10^{2} B_{T}(T)\left(Z / \tau_{i}\right)\left(n \sigma_{*}\right)^{-3 / 2}} \\
& \quad \times \sqrt{2\left(1+\tau_{i} / Z\right) / A_{i} R} .
\end{aligned}
$$

We remark here that the existence of threshold edge electron temperature for $\mathrm{L}-\mathrm{H}$ transition $\left(T_{e, c r i t}\right)$ has been observed in numerous tokamak experiments. ${ }^{25,38,39}$ Since $T_{e, c r i t}$ is a natural consequence of the turbulence quench rule by mean $E \times B$ shear in our model, this suggests that the onset of positive feedback by the mean $E \times B$ shear will be the possible origin of $T_{e, c r i t}$ in L-H transition.

The combination of this requirement with the power balance relation yields an expression for the $\mathrm{L}-\mathrm{H}$ transition power threshold

$$
P_{t h}(\mathrm{MW}) \geq P_{t u r b}(\mathrm{MW})+P_{r a d}(\mathrm{MW}),
$$

where

$$
\begin{aligned}
P_{\text {turb }}(\mathrm{MW})= & 0.075 C_{T E P} \frac{Z\left(1+\tau_{i} / Z\right)^{3 / 2}}{\tau_{i} A_{i}^{1 / 2}}\left(n \sigma_{*}\right)^{1 / 2} \\
& \times\left[1+\frac{1}{36 n \sigma_{*} L_{T}}\right] \times B_{T} R^{1 / 2} S,
\end{aligned}
$$

and $P_{\text {rad }}$ is given in Eq. (4). In Eq. (12), $n$ and $\sigma *$ are normalized to $10^{20} \mathrm{~m}^{-3}$ and $3.6 \times 10^{-19} \mathrm{~m}^{2}$, respectively.

Equation (11) is the principal result of this work. Combined with Eqs. (4) and (12), it gives the power threshold required for $\mathrm{L}-\mathrm{H}$ transition in tokamaks under the assumption of high- $m$ DRBM dominant turbulent transport prior to L-H transition. The coefficient $C_{T E P}$, representing the TEP effect, is treated as an empirical parameter, the value of which is to be determined by the comparison between calculated $P_{t h}$ using Eq. (11) and experimental results.

From Eq. (12), one can find that $P_{\text {turb }}$ scales as $P_{\text {turb }} \propto\left(n \sigma_{*}\right)^{1 / 2}+\left(1 / 36 \Delta_{R}\right)\left(n \sigma_{*}\right)^{-1 / 2}$. The origin of the existence of the roll-over density is then obvious: it comes from the turbulent conduction inside EDL, which is proportional to $\left(n \sigma_{*}\right)^{-1 / 2}$. This constitutes a dominant power loss channel in low density regime where convective $\left(\propto\left(n \sigma_{*}\right)^{1 / 2}\right)$ and radiative $\left(\propto n^{2}\right)$ losses become small. Thus, we identify that the "U"-shaped non-monotonic density scaling in $P_{t h}$ originates from the rapid increase of density scaling length, $L_{n}=1 / n \sigma_{*}$, as density becomes low.

In our model, the turbulent conductive loss is dominant in the low density regime, while radiative loss becomes predominant in the high density regime. The turbulent convective loss is typically much smaller than the radiative loss when $n\left(10^{20} \mathrm{~m}^{-3}\right) \geq 0.15$.

The roll-over density is then determined by the balance between the conductive and radiative losses in low and high density regimes, respectively. One can obtain the scaling of the roll-over density in $P_{t h}$ vs. $n$ from $\partial P_{t h} / \partial n=0$, giving rise to

$$
n_{\min } \approx \Delta_{R}^{-0.8}\left[\frac{B_{T}}{\tau_{i} f_{Z} R_{T}}\right]^{0.4}\left[\frac{Z^{2} R}{\sigma_{*} A_{i}}\right]^{0.2} .
$$

The relation $n_{\min } \propto \Delta_{R}^{-0.8}$ in Eq. (13) implies that the EDL width is the most influential discharge condition to determine $n_{\text {min }}$. In addition to $\Delta_{R}, B_{T}$ is also an important external factor to determine $n_{\min }$ for given discharge parameters, such as $\Delta_{R}$ and impurity contents/levels. So, one expects higher roll-over density as $B_{T}$ increases, which qualitatively agrees with experiments. However, the $B_{T}$ scaling of $n_{\min }$ is relatively weak compared to the experimentally inferred scaling, $n_{\min } \propto B_{T}$. If we include the convective loss term, however, $\partial P_{t h} / \partial n=0$ is reduced to the fifth order algebraic equation. Then, $n_{\min }$ shows a stronger $B_{T}$ scaling, approaching to $n_{\min } \propto B_{T}^{2 / 3}$. The isotope scaling to $n_{\min }$, $n_{\text {min }} \propto\left(Z^{2} / A_{i}\right)^{0.2}$, is marginal.

It is instructive to look into Eq. (12) in terms of power threshold scaling. When $P_{t h}$ is written in terms of a power law for density, magnetic field, and major radius, i.e., $P_{t h}=C \bar{n}^{X} B_{T}^{Y} R^{Z}$, the exponents of them are known to satisfy 
the Kadomtsev constraint, $8 X+5 Y-4 Z=3 .^{2,40}$ Atomic physics processes, such as radiation, has not been considered to derive the constraint, while experiments show a clear dependence of $P_{t h}$ on wall conditioning and edge neutral density. So, it is appropriate to examine if $P_{\text {turb }}$ given in Eq. (12) satisfy the Kadomtsev constraint (i.e., not taking radiative power loss which definitely violate the constraint). Assuming a linear relation of $n$ to $\bar{n}$ as observed in recent Alcator C-Mod experiment ${ }^{14}$ and taking $1 / n \sigma_{*} L_{T}$ as the ratio, $L_{n} / L_{T}$, one can obtain

$$
P_{\text {turb }} \propto \bar{n}^{0.5} B_{T} R^{1.5} .
$$

It is easy to see that Eq. (14) satisfies the Kadomtsev constraint. In fact, we found a remarkable coincidence between Eq. (14) and the empirical power threshold expression in Ref. 2, $P_{t h}=C B_{T} \bar{n}^{0.75} R^{2}\left(\bar{n} R^{2}\right)^{\alpha}$ with $-0.25 \leq \alpha \leq 0.25$ and $C=(0.45 \pm 0.1) \times 0.6^{\alpha}$ (Ref. 2$)$. The latter exactly reduces to Eq. (14) when $\alpha=-0.25$, with the range of $C$ value, $0.308 \leq C \leq 0.484$. Reference 2 suggests that the major source of uncertainty of the power threshold prediction for ITER comes from the uncertainty in major radius scaling, $P_{t h} \propto R^{Z}$, where $1.5 \leq Z \leq 2.5 .^{2}$ Since $P_{t h} \propto R^{1.5}$ in our model, which is the lowest exponent for the given range, our model gives a favorable prediction for ITER power threshold, as will be discussed in Sec. III.

Regarding the isotope and atomic charge scaling, Eq. (12) indicates that $P_{t h}$ is proportional to $Z\left(1+\tau_{i} / Z\right)^{3 / 2} / A_{i}^{1 / 2}$. Then, in similar discharge conditions with $\tau_{i} \approx 1, P_{t h}$ for deuterium discharges is $\sqrt{2}$ times lower than that of hydrogen discharges. This is weaker than the scaling, $P_{t h} \propto 1 / A_{i}$. Equation (12) also indicates that $P_{t h}$ for helium discharges with $\mathrm{He}_{4}^{2}$ as a working gas will be $3 \sqrt{3} / 8 \simeq 0.65$ times lower than that of hydrogen. If we take $\sigma_{*}$ into account, however, the actual ratio of $P_{t h}$ for deuterium and He discharges will be a function of the ratio $\hat{\sigma}=\sigma_{*}(H e) / \sigma_{*}(D)$, which is a complicated function of electron temperature and density. Typically, $\hat{\sigma}$ is lower than 1 . This may explain why $P_{t h}$ for He discharges is between those of hydrogen and deuterium, depending on discharge conditions such as wall conditioning method, edge electron temperature and density. ${ }^{17}$

\section{APPLICATION TO PRESENT DEVICES AND ITER}

To calculate $P_{t h}$ using Eqs. (4), (11), and (12), it is necessary to determine the coefficient $C_{T E P}$. At present, there is no generally accepted theory basis to determine $C_{T E P}$ from first principles. So, we take an empirical approach in this study. Namely, we evaluate $C_{T E P}$ from experimental data and treat it as a fixed coefficient to calculate $P_{t h}$. In this work, we estimate $C_{T E P}$ using the KSTAR L-H transition data published in Ref. 12. Table I summarizes numerical values for various plasma parameters in Eqs. (4) and (12) that are used to calculate $P_{t h}$. We note that some parameters, such as $\Delta_{R}, f_{z}$, and $R_{T}$, are not available in the literature. Thus, we assumed typical values for these parameters, which are expected in KSTAR experiments.

Figure 1 shows turbulent and radiative (red and blue dashed lines, respectively) power losses, and $P_{t h}$ (black solid
TABLE I. Parameters being used to calculate $P_{t h}$ in various tokamaks.

\begin{tabular}{lcccc}
\hline \hline Parameter & KSTAR $^{12}$ & ASDEX-U $^{13}$ & JET $^{15}$ & ITER $^{41}$ \\
\hline Working gas & $D$ & $\mathrm{D}$ & $\mathrm{D}$ & $\mathrm{D}, \mathrm{H}$ \\
$B_{T}(\mathrm{~T})$ & 2.0 & 2.3 & 2.7 & 5.3 \\
$R(\mathrm{~m})$ & 1.8 & 1.63 & 2.96 & 6.2 \\
$a(\mathrm{~m})$ & 0.5 & 0.5 & 1.25 & 2.0 \\
$\kappa$ & 1.8 & 1.7 & 1.7 & 1.7 \\
$S\left(\mathrm{~m}^{2}\right)$ & 51.7 & 44.9 & 203.7 & 682.7 \\
$\tau_{i}=T_{i} / T_{e}$ & 1.0 & 1.0 & 1.0 & 1.0 \\
$\sigma *\left(3.6 \times 10^{-19} \mathrm{~m}^{-2}\right)$ & 1.0 & 1.0 & 1.0 & 1.0 \\
$f_{Z}\left(10^{-2}\right)$ & 10 & 10 & 10 & 5.0 \\
$R_{T}\left(10^{-39} \mathrm{MWm}^{3}\right)$ & 20 & 15 & 7.0 & 10.0 \\
$\Delta_{R} / a$ & 0.05 & 0.02 & 0.03 & 0.05 \\
\hline \hline
\end{tabular}

line) as a function of edge density. The lower triangles in Fig. 1 represent selected experimental data points reproduced from Ref. 12. For comparison, we also show $P_{t h}$ evaluated from the empirical scaling law, Eq. (1), in a green solid line. We found that $C_{T E P}=0.008$ (i.e., $0.89 \times 10^{-19} \mathrm{~m}^{-3} / \mathrm{T}$ ) gives a good agreement with experimental data, as can be seen in Fig. 1. Of course, care should be taken of the validity of this number because it is obtained by using some assumed values as discussed earlier. Nonetheless, $C_{T E P}=0.008$ gives a reasonable agreement with experimental data for other devices, as will be shown shortly. Thus, we use $C_{T E P}=0.008$ to calculate $P_{t h}$ for other devices and ITER in this study.

As discussed in Sec. II, turbulent transport is a dominant loss channel in low density regime $(n \leq 0.17)$, while radiative loss prevails in relatively high density regime $(n \geq 0.17)$. One can clearly see the existence of the roll-over density around $n$ $\simeq 0.1$. This corresponds to $\bar{n}=0.2$, if we assume $n=0.5 \bar{n}$ as observed in recent Alcator C-Mod experiments. ${ }^{14} P_{t h}$ shown in Fig. 1 (using parameters in Table I and $C_{T E P}$ evaluated from experimental data) agrees well with experimental results reported in Ref. 12, while that calculated from the

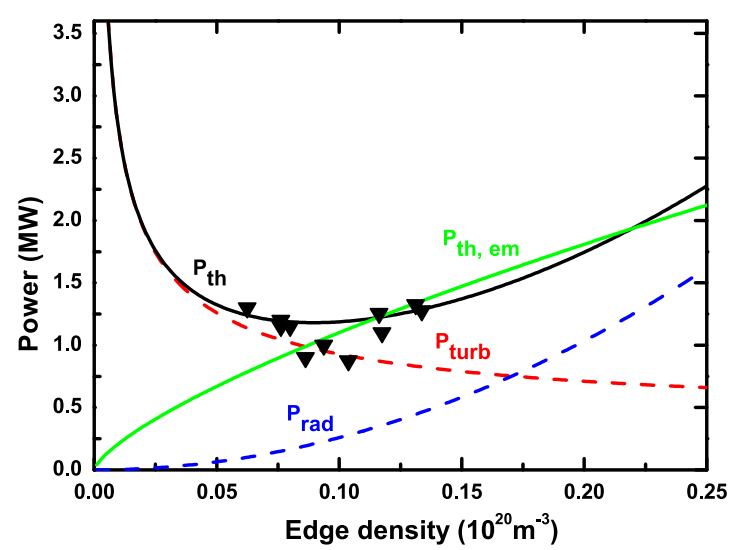

FIG. 1. Turbulent $\left(P_{\text {turb }}\right.$, red dashed) and radiative $\left(P_{\text {rad }}\right.$, blue dashed) power through a narrow edge dissipation layer as a function of edge density. Analytic $\left(P_{t h}=P_{\text {turb }}+P_{\text {rad }}\right)$ and empirical $\left[P_{t h, e m}\right.$, Eq. (1)] L-H transition power threshold are shown in black and green solid lines, respectively. Lower triangles denote some data points reproduced from Ref. 12. The TEP coefficient $\left(C_{T E P}\right)$ is obtained by fitting the data points using the KSTAR parameters summarized in Table I. Semi-analytic $P_{\text {th }}$ shows a good agreement with experimental results, while empirical one shows a considerable deviation at low density regime. 
empirical scaling law shows a deviation in the low density regime. Such a discrepancy in $P_{t h}$ between the empirical scaling and experimental data in the low density regime has been also reported in some tokamaks. ${ }^{13-15}$

It is of importance to perform a sensitivity study of the $P_{\text {th }}$ curve to discharge parameters since we used some assumed values for them to obtain $C_{T E P}$. Among the parameters, the width of EDL, $\Delta_{R}$, has most significant influence in $P_{\text {th }}$ in the low density regime, as shown in Fig. 2(a). Not only $P_{t h}$ but also $n_{\text {min }}$ is considerably affected by $\Delta_{R}$. Low $\Delta_{R}$ brings about the flattening of the minimum $P_{t h}$ density, i.e., increasing the roll-over density. In contrast to this, the impact of $P_{\text {rad }}$ on $P_{\text {th }}$ in the low density regime due to the uncertainties of $f_{Z}$ and volumetric radiation $\left(R_{T}\right)$ is marginal. The change of $f_{Z}$ and $R_{T}$ has little influence on $P_{t h}$ in the low density regime $(n \leq 0.15)$, as shown in Fig. 2(b), where $P_{t h}$ vs. $n$ curves are plotted for three values of $f_{Z}\left(f_{Z}=5.0,10.0\right.$, and 15.0 corresponding to red, green, black, and blue lines, respectively). Low radiation loss also gives rise to the flattening of minimum $P_{t h}$ density. Increase of $f_{Z}$ brings about the increase of the slope of $P_{t h}$ (i.e., increase of $P_{t h}$ ) in high density regime resulting in further proximity to the characteristic U-shape in a $P_{t h}$ vs. $n$ curve.

Next, we apply our model to existing tokamak devices for which published data for $\mathrm{L}-\mathrm{H}$ transition are available. We have chosen two devices for this study: ASDEX-U ${ }^{13}$ and JET. ${ }^{15}$ Figures 3(a) and 3(b) show the results of $P_{t h}$

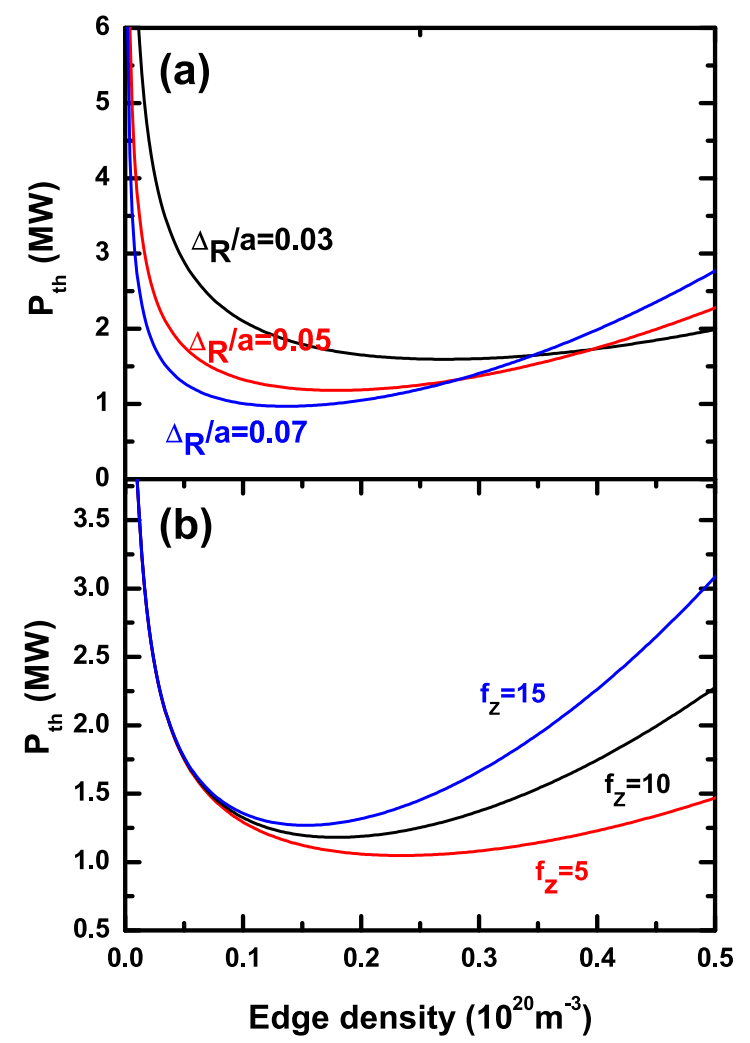

FIG. 2. Sensitivity of $P_{\text {th }}$ to the (a) ratio of EDL width $\left(\Delta_{R}\right)$ to minor radius (a) and (b) impurity fraction $\left(f_{Z}\right) . \Delta_{R}$ has a significant influence on $P_{t h}$ in our model, in particular, in the low density regime. Higher $f_{Z}$ results in the proximity of a characteristic "U"-shape curve due to the increase of $P_{\text {rad }}$ in the high density regime without affecting $P_{t h}$ significantly in the low density regime. calculations for ASDEX-U and JET, respectively. In these calculations, we use $C_{T E P}=0.008$ which has been obtained from KSTAR experimental data. Plasma parameters being used to obtain Fig. 3 are also given in Table I. Again, lower triangles in Figs. 3(a) and 3(b) denote selected experimental data points from Refs. 13 and 15, respectively. Both show familiar "U"-shaped curves with good agreements with experimental data. In Fig. 3(b), we show only data points for MkIIGB Septum configuration at JET exhibiting a clear density roll-over in $P_{t h}$. We mention that no density roll-over is observed when septum is removed (SRP configuration).

Although not presented here, we remark that our model does not fit quite well with Alcator C-Mod data presented in Ref. 14. The roll-over density matches well if we use $\Delta_{R} / a=0.01$, but published $P_{t h}$ in Ref. 14 is $50 \%-100 \%$ higher than that predicted by the model over a wide range of density. Disparity in roll-over density between Alcator C-Mod and other devices has been already recognized in previous works. ${ }^{2,11}$ The physics origin behind this discrepancy is unclear yet.

Having assessed the validity of the model to existing data, we apply our model to predict $P_{t h}$ in ITER. Figure 4(a) shows predicted $P_{t h}$ for ITER hydrogen (blue line) and deuterium (black line) discharges. We used typical ITER plasma parameters summarized in Table I. For comparison, we also present $P_{t h}$ obtained from the empirical scaling in blue line. $P_{t h}$ VS. $n$ curves in ITER also exhibit an U-shaped feature with a roll-over density around $n \sim 0.1\left(10^{20} \mathrm{~m}^{-3}\right)$. When $n \geq n_{\text {min }}$, our model predicts lower $P_{t h}$ than the empirical scaling law for low density, while it does higher one for high density $(n \geq 0.4)$. If we assume $n=0.5 \bar{n}$ as in Fig. $1, P_{t h}$ for $\bar{n}=$ $0.5 \times 10^{20} \mathrm{~m}^{-3}$ and $1.0 \times 10^{20} \mathrm{~m}^{-3}$ are 37.3 and $101.9 \mathrm{MW}$, respectively. Both values are within the $95 \%$ confidence interval of the empirical scaling law. ${ }^{11}$ So, accessing $\mathrm{H}$-mode at low density $\mathrm{He}$ discharges should be readily achieved with the power level available in the initial phase of ITER operation, while it is problematic at high density.
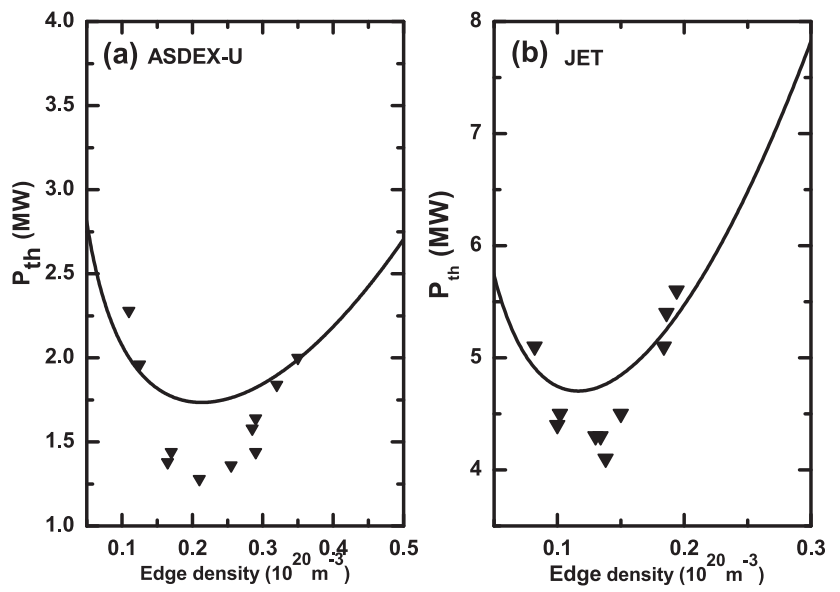

FIG. 3. Calculations of $P_{\text {th }}$ for (a) ASDEX-U and (b) JET. Plasma parameters summarized in Table I and $C_{T E P}=0.008$ have been used. Lower triangles denote some data points reproduced from Ref. 13, for ASDEX-U, and 15 , for JET, respectively. Semi-analytic $P_{t h}$ shows reasonable agreements with experimental data for both devices, except near the roll-over density. Only data points for MkIIGB Septum configuration are shown in (b) exhibiting a clear density roll-over in $P_{t h}$. 

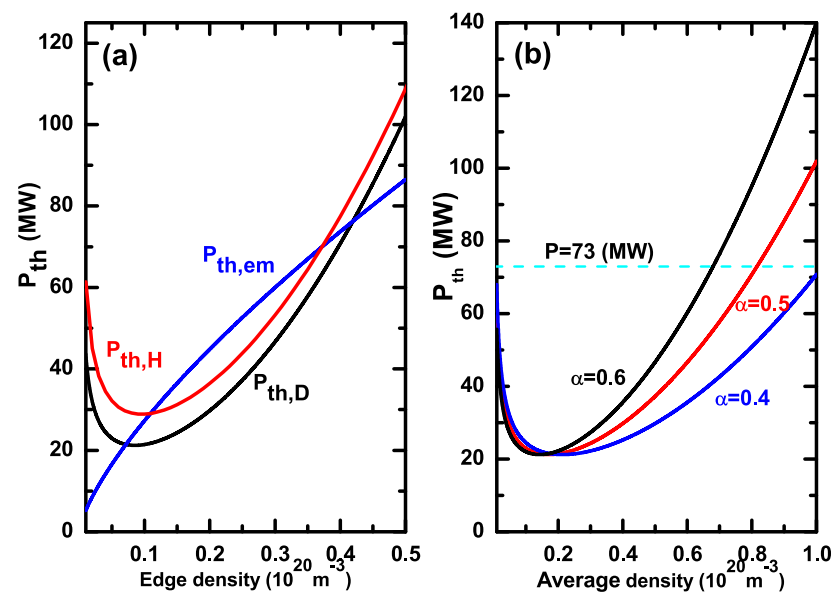

FIG. 4. (a) Predictions of $P_{t h}$ for ITER hydrogen $\left(P_{t h, H}\right.$, red solid line) and deuterium $\left(P_{t h, D}\right.$, black solid line) as functions of edge density. $P_{t h}$ from the empirical scaling law, Eq. (1), is shown in blue solid line. ITER plasma parameters summarized in Table I have been used. (b) $P_{t h}$ vs. average density $(\bar{n})$ for various ratio of edge density $(n)$ to $\bar{n}, f_{n}=n / \bar{n}$. A linear relationship between $n$ and $\bar{n}$ is assumed. Cyan dashed line indicates auxiliary heating power at initial operation of ITER $\left(P_{\text {aux }}=73 \mathrm{MW}\right)$. Decrease of $f_{n}$ significantly reduces $P_{t h}$ at ITER target operation density, $\bar{n} \approx 0.9$. When $\alpha \leq 0.45$, $P_{\text {th }}$ is less than $P_{\text {aux }}$.

The current envision of accessing a high density Hmode in ITER is to exploit hysteresis in power, i.e., accessing H-mode at low density and ensuing density ramp up. In this case, we may define the "strength" of hysteresis as $\left(n_{\max }-n_{0}\right) / n_{0}$, where $n_{0}$ is the density at which L-H transition occurs with a given power, $P_{0}$ and $n_{\text {max }}$ is the maximum density that can maintain the $\mathrm{H}$-mode state with $P_{0}$. The physics basis determining the hysteresis strength for given $P_{0}$ has not been established yet. Given the lack of physics understanding, it will be necessary to reduce expected $P_{t h}$ at high density to ensure high density $\mathrm{H}$-mode operation in ITER. If $P_{\text {max }} / P_{0}\left(P_{\text {max }}\right.$ is the power threshold at $\left.n=n_{\text {max }}\right)$ is too high, we may not ensure the effect of hysteresis to maintain the H-mode. In our model, $P_{t h}$ strongly depends on density $\left(P_{t h} \propto n^{2}\right)$ at high density due to the radiative loss, so, the hysteresis effect might be questionable to sustain the $\mathrm{H}$-mode state. The most effective way of doing so is to reduce the edge density and radiative losses.

Figure 4(b) shows $P_{t h}$ vs. line averaged density $(\bar{n})$ for three different ratios of edge density to average density, $f_{n}=n / \bar{n}$. Here, we assume a linear relationship between $n$ and $\bar{n}$. One can see the reduction of $P_{t h}$ at high density when $f_{n}$ decrease. In fact, we found that $P_{t h}$ is less than the heating power planned in the initial phase of ITER operation (73 MW) when $f_{n} \leq 0.45$. The reduction of edge density may be achieved by using deep pellet injection as a main fuelling tool. In this regard, it is of interest to study high density L-H transition with deep pellet fuelling and comparison of $P_{t h}$ with the gas-puffed case.

\section{SUMMARY AND CONCLUSIONS}

In summary, we presented a model for L-H transition power threshold $\left(P_{t h}\right)$ in tokamaks, based on a simple edge power balance relation. Turbulent heat transport and radiative losses at a narrow edge layer are assumed to be responsible for the heat disposal coming out of a tokamak core region. Two assumptions have been made in our analytic study:

- High- $m$ DRBM is the dominant turbulence driver in tokamak edge plasmas.

- The pre-transition L-mode profile and turbulent diffusivity pertain to turbulent equipartition.

Then, we derived $P_{t h}$ as a function of plasma parameters prior to L-H transition by exploiting the turbulence quench rule by mean $E \times B$ shear. Our model leaves an undetermined coefficient, the TEP coefficient $C_{T E P}$ whose value has been obtained by comparison with experimental data (in this work, using the published KSTAR experimental data in Ref. 12). Our model reproduces features of the $P_{t h}$ vs. density curves observed in present-day tokamaks. In particular, it exhibits the characteristic feature of an "U"-shaped $P_{t h}$ vs. $n$ curve, indicating the existence of roll-over density below which $P_{t h}$ increases as density decreases. It turns out that this is due to the increase of the density scaling length in the low density regime. This interpretation is conceptually disparate with that of a recently published article where the two-point scrape off layer (SOL) transport model has been employed to evaluate $P_{t h}{ }^{42}$ We found that our turbulent transport model satisfies the Kadomtsev constraint and reproduces the empirical scaling law given in Ref. 2.

Application of our model to existing tokamak devices shows a reasonable agreement with experimental results. An exception for this relatively high degree of agreements is the Alcator C-Mod tokamak in which the disparity of minimum power threshold density between other machines has been recognized in earlier works. ${ }^{2,11}$ The physical origin behind this discrepancy is unclear. Our model was applied to predict $P_{t h}$ in ITER. Large radiation loss at high density $\left(n \geq 0.35 \times 10^{20} \mathrm{~m}^{-3}\right)$ makes dubious to access high density H-mode in ITER by the exploitation of hysteresis, given the limited power level in the initial ITER operation phase. To avoid this difficulty, we highlighted the importance of reducing edge density by deep pellet injection to reduce $P_{t h}$ at high density. A comparative study of L-H transition by varying edge density will be a good test-bed for the validation of our model.

Finally, we remark that our work should be regarded as a semi-analytic approach bridging the gap between empirical scaling and rigorous derivation of $P_{L H}$ from microphysics. The latter, of course, is the ultimate goal of theoretical endeavour, which is still far from the realization due to the complication of tokamak edge turbulence. A natural next step will then be the consideration of power balance in the presence of general drift wave turbulence. A way to identify key dynamical processes involved in L-H transition is to perform fully nonlinear simulations. However, performing fluxdriven, global nonlinear simulations using the first principlebased code is a challenging task. In this regard, dynamic flux-tube simulations are interesting in elucidating the effects of local edge conditions, such as edge temperature and density, on L-H transition and $P_{L H}$. Flux-tube simulations are not computationally demanding compared to global simulations. Further, the effects of other instabilities (such as ion 
temperature gradient/trapped electron modes) that have not considered in our study can be easily incorporated in these simulations. This is left as a future study.

\section{ACKNOWLEDGMENTS}

One of the authors (R. Singh) thanks the ITER Organization for their support while this work was initiated. This research was supported by the World Class Institute (WCI) Program of the National Research Foundation (NRF) funded by the Ministry of Science, ICT and Future Planning (MSIP) of Korea (WCI 2009-001). The views and opinions expressed herein do not necessarily reflect those of the ITER Organization.

${ }^{1}$ F. Wagner, G. Becker, K. Behringer, D. Campbell, A. Eberhagen, W. Engelhardt, G. Fussmann, O. Gehre, J. Gernhardt, G. v. Gierke, G. Haas, M. Huang, F. Karger, M. Keilhacker, O. Klueber, M. Kornherr, K. Lackner, G. Lisitano, G. G. Lister, H. M. Mayer, D. Meisel, E. R. Mueller, H. Murmann, H. Niedermeyer, W. Poschenrieder, H. Rapp, H. Rohr, F. Schneider, G. Siller, E. Speth, A. Staebler, K. H. Steuer, G. Venus, O. Vollmer, and Z. Yue, Phys. Rev. Lett. 49, 1408 (1982).

${ }^{2}$ ITER Physics Expert Groups on Confinement and Transport and Confinement Modelling and Database, Nucl. Fusion 39, 2175 (1999).

${ }^{3}$ J. W. Connor and H. R. Wilson, Plasma Phys. Controlled Fusion 42, R1 (2000).

${ }^{4}$ H. Biglari, P. H. Diamond, and P. W. Terry, Phys. Fluids B 2, 1 (1990).

${ }^{5}$ T. S. Hahm and K. H. Burrell, Phys. Plasmas 2, 1648 (1995).

${ }^{6}$ A. Hasegawa and M. Wakatani, Phys. Rev. Lett. 59, 1581 (1987).

${ }^{7}$ P. H. Diamond, S.-I. Itoh, K. Itoh, and T. S. Hahm, Plasma Phys. Controlled Fusion 47, R35 (2005).

${ }^{8}$ K. Miki, P. H. Diamond, Ö. D. Gürcan, G. R. Tynan, T. Estrada, L. Schmitz, and G. S. Xu, Phys. Plasmas 19, 092306 (2012).

${ }^{9}$ L. Schmitz, L. Zeng, T. L. Rhodes, J. C. Hillesheim, E. J. Doyle, R. J. Groebner, W. A. Peebles, K. H. Burrell, and G. Wang, Phys. Rev. Lett. 108, 155002 (2012).

${ }^{10}$ T. Estrada, C. Hidalgo, T. Happel, and P. H. Diamond, Phys. Rev. Lett. 107, 245004 (2011).

${ }^{11}$ Y. R. Martin, T. Takizuka, and ITPA CDBM H-mode Threshold Database Working Group, J. Phys.: Conf. Ser. 123, 012033 (2008).

${ }^{12}$ J.-W. Ahn, H.-S. Kim, Y. S. Park, L. Terzolo, W. H. Ko, J.-K. Park, A. C. England, S. W. Yoon, Y. M. Jeon, S. A. Sabbagh, Y. S. Bae, J. G. Bak, S. H. Hahn, D. L. Hillis, J. Kim, W. C. Kim, J. G. Kwak, K. D. Lee, Y. S. Na, Y. U. Nam, Y. K. Oh, and S. I. Park, Nucl. Fusion 52, 114001 (2012).

${ }^{13}$ F. Ryter, T. Pütterich, M. Reich, A. Scarabosio, E. Wolfrum, R. Fischer, M. G. Adamov, N. Hicks, B. Kurzan, C. Maggi, R. Neu, V. Rohde, G. Tardini, and ASDEX Upgrade Team, Nucl. Fusion 49, 062003 (2009).

${ }^{14}$ Y. Ma, J. W. Hughes, A. E. Hubbard, B. LaBombard, R. M. Churchill, T. Golfinopolous, N. Tsujii, and E. S. Marmar, Nucl. Fusion 52, 023010 (2012).

${ }^{15}$ Y. Andrew, R. Sartori, E. Righi, E. de la Luna, S. Hacquin, D. F. Howell, N. C. Hawkes, L. D. Horton, A. Huber, A. Korotkov, M. G. O'Mullane, and JET-EFDA Contributors, Plasma Phys. Controlled Fusion 48, 479 (2006).

${ }^{16}$ E. Righi, D. V. Bartlett, J. P. Christiansen, G. D. Conway, J. G. Cordey, L.-G. Eriksson, H. P. L. De Esch, G. M. Fishpool, C. W. Gowers, J. C. M. de Haas, P. J. Harbour, N. C. Hawkes, J. Jacquinot, T. T. C. Jones, W. Kerner, Q. A. King, C. G. Lowry, R. D. Monk, P. Nielsen, F. G. Rimini, G. Saibene, R. Sartori, B. Schunke, A. C. C. Sips, R. J. Smith, M. F.
Stamp, D. F. H. Start, K. Thomsen, B. J. D. Tubbing, and N. Zornig, Nucl. Fusion 39, 309 (1999).

${ }^{17}$ P. Gohil, D. Battaglia, E. de la Luna, M. E. Fenstermacher, J. Hughes, S. Kaye, A. Kirk, Y. Ma, R. Maingi, Y. Martin, D. C. McDonald, H. Meyer, F. Ryter, the ITPA Topical Groups on Transport and Confinement and Pedestal and Edge Physics, and the Research Teams of ASDEX Upgrade, C-Mod, DIII-D, EFDA-JET, MAST and NSTX, in Proceedings of the 24th IAEA Fusion Energy Conference, San Diego, US (IAEA, Vienna, 2012), ITR/P1-36.

${ }^{18} \mathrm{~S}$. Chandrasekhar, An Introduction to the Study of Stellar Structure (Dover Publications, New York, NY, 1958), Chap. 5.

${ }^{19}$ P. N. Guzdar, J. F. Drake, D. McCarthy, A. B. Hassam, and C. S. Liu, Phys. Fluids B 5, 3712 (1993).

${ }^{20}$ S. V. Novakovskii, P. N. Guzdar, J. F. Drake, C. S. Liu, and F. L. Waelbroeck, Phys. Plasmas 2, 781 (1995).

${ }^{21}$ B. N. Rogers, J. F. Drake, and A. Zeiler, Phys. Rev. Lett. 81, 4396 (1998).

${ }^{22}$ R. Singh, V. Tangri, P. K. Kaw, and P. N. Guzdar, Phys. Plasmas 12, 092307 (2005).

${ }^{23}$ J. R. Myra, D. A. D’Ippolito, X. Q. Xu, and R. H. Cohen, Phys. Plasmas 7, $4622(2000)$

${ }^{24}$ C. Bourdelle, X. Garbet, R. Singh, and L. Schmitz, Plasma Phys. Controlled Fusion 54, 115003 (2012).

${ }^{25}$ A. E. Hubbard, R. L. Boivin, J. F. Drake, M. Greenwald, Y. In, J. H. Irby, B. N. Rogers, and J. A. Snipes, Plasma Phys. Controlled Fusion 40, 689 (1998).

${ }^{26}$ J. L. Terry, S. J. Zweben, K. Hallatschek, B. LaBombard, R. J. Maqueda, B. Bai, C. J. Boswell, M. Greenwald, D. Kopon, W. M. Nevins, C. S. Pitcher, B. N. Rogers, D. P. Stotler, and X. Q. Xu, Phys. Plasmas 10, 1739 (2003).

${ }^{27}$ M. A. Mahdavi, R. Maingi, R. J. Groebner, A. W. Leonard, T. H. Osborne, and G. Porter, Phys. Plasmas 10, 3984 (2003).

${ }^{28}$ R. J. Groebner, M. A. Mahdavi, A. W. Leonard, T. H. Osborne, N. S. Wolf, G. D. Porter, P. C. Stangeby, N. S. Brooks, R. J. Colchin, and L. W. Owen, Nucl. Fusion 44, 204 (2004).

${ }^{29}$ W. M. Stacey and R. J. Groebner, Phys. Plasmas 13, 012513 (2006).

${ }^{30}$ M. Z. Tokar, Phys. Rev. Lett. 91, 095001 (2003).

${ }^{31}$ J. Wesson, Tokamaks, 2nd ed. (Oxford University Press, Oxford, NY, 1997), p. 506.

${ }^{32}$ K. H. Burrell, E. J. Doyle, P. Gohil, R. J. Groebner, J. Kim, R. J. La Haye, L. L. Lao, R. A. Moyer, T. H. Osborne, W. A. Peebles, C. L. Rettig, T. H. Rhodes, and D. M. Thomas, Phys. Plasmas 1, 1536 (1994).

${ }^{33}$ K. H. Burrell, Phys. Plasmas 6, 4418 (1999).

${ }^{34}$ E. J. Kim and P. H. Diamond, Phys. Rev. Lett. 90, 185006 (2003).

${ }^{35}$ J. Nycander and V. V. Yankov, Phys. Plasmas 2, 2874 (1995).

${ }^{36}$ V. Naulin, J. Nycander, and J. J. Rasmussen, Phys. Rev. Lett. 81, 4148 (1998).

${ }^{37}$ M. B. Ishichenko, A. V. Gruzinov, and P. H. Diamond, Phys. Rev. Lett. 74, 4436 (1995).

${ }^{38}$ W. Suttrop, M. Kaufmann, H. J. de Blank, B. Brusehaber, K. Lackner, V. Mertens, H. Murmann, J. Neuhauser, F. Ryter, H. Salzmann, J. Schweinzer, J. Stober, H. Zohm, and the ASDEX Upgrade Team, Plasma Phys. Controlled Fusion 39, 2051 (1997).

${ }^{39}$ E. Righi, D. Bartlett, G. Conway, J. G. Cordey, L.-G. Eriksson, C. Gormezano, J. C. M. de Haas, L. Horton, J. Jacquinot, C. Lowry, G. Saibene, R. Sartori, M. Stamp, D. F. H. Start, and K. Thomsen, Plasma Phys. Controlled Fusion 40, 721 (1998).

${ }^{40} \mathrm{~F}$. Ryter and the H-mode Database Working Group, Plasma Phys. Controlled Fusion 38, 1279 (1996).

${ }^{41}$ A. R. Polevoi, M. Shimada, M. Sugihara, Yu. L. Igitkhanov, V. S. Mukhovatov, A. S. Kukushkin, S. Yu. Medvedev, A. V. Zvonkov, and A. A. Ivanov, Nucl. Fusion 45, 1451 (2005).

${ }^{42}$ W. Fundamenski, F. Militello, D. Moulton, and D. C. McDonald, Nucl. Fusion 52, 062003 (2012). 